\title{
Isotope effects in the vibrational deactivation of large molecules
}

\author{
Beatriz M. Tosellia) and John R. Barker \\ Department of Atmospheric, Oceanic, and Space Sciences, Department of Chemistry, Space Physics \\ Research Laboratory, The University of Michigan, Ann Arbor, Michigan 48109-2143
}

(Received 9 March 1992; accepted 20 April 1992)

\begin{abstract}
Collisional deactivation of highly vibrationally excited gas phase toluene- $d_{8}$ and benzene- $d_{6}$ pumped at $248 \mathrm{~nm}$, has been investigated by monitoring the time resolved infrared fluorescence from the C-D stretch modes near $4.3 \mu \mathrm{m}$. For toluene- $d_{8}$, energy transfer data were obtained for about 20 collider gases, including unexcited toluene- $d_{8}$; for benzene- $d_{6}$, only a few colliders were investigated. For both systems the data were analyzed by an inversion technique that converts the fluorescence decay to the bulk average energy, from which is calculated the average energy transferred per collision, $\langle\langle\Delta E\rangle\rangle_{\mathrm{inv}}$. Data obtained earlier for benzene- $d_{0}$ were reanalyzed and the revised results are reported. Results for both normal and deuterated excited species show $\langle\langle\Delta E\rangle\rangle_{\text {inv }}$ to be nearly directly proportional to the vibrational energy $\langle\langle E\rangle\rangle_{\mathrm{inv}}$ of the excited molecule from 5000 to $25000 \mathrm{~cm}^{-1}$. However, for pure toluene- $d_{8}$, benzene- $d_{6}$, and a few other collider gases at high energies, the slope of the $\langle\langle\Delta E\rangle\rangle_{\text {inv }}$ vs $\langle\langle E\rangle\rangle_{\text {inv }}$ curve is reduced and even becomes negative at sufficiently high energies. The results obtained for normal and deuterium-containing species are discussed in terms of possible quantum effects and mechanisms for energy transfer. In particular, it is considered likely that $V-T / R$ energy transfer dominates over $V-V$, and the lowest frequency vibrational modes are the conduits for the energy transfer, in agreement with results for small molecules. Attention is called to a fundamental difference between classical and quantum statistics and how this difference may adversely affect classical trajectory simulations of large molecules.
\end{abstract}

\section{INTRODUCTION}

Isotopic substitution alters some molecular propertics more than others. The potential energy surface is not affected as long as the Born-Oppenheimer approximation is valid, which is expected to be the case for the ground electronic states of the species investigated here. Vibrational frequencies and moments of inertia are affected to a greater, or lesser extent, depending on the vibrational modes and on the geometry of the molecule. If the symmetry of the molecule remains unchanged by the substitution, the dipole selection rules remain the same and it is possible that energy transfer "propensity rules" also remain unchanged. However, the magnitude of possible quantum effects will be affected by the changes in mass introduced by isotopic substitution. Thus isotopic substitution may provide a means to determine some of the mechanisms by which vibrational deactivation of large molecules occurs, but it is not a perfect tool, because several molecular properties are changed simultaneously and disentangling the various effects may be difficult.

Collisional deactivation of highly excited polyatomics has been the subject of much study and although energytransfer data are now available from many experiments, ${ }^{7}$ quantitative understanding of the process is still poor. Several experimental techniques have bcen used recently to study energy transfer in midsized and large polyatomic molecules. The time resolved infrared fluorescence technique (IRF) technique has been used to measure the average energy

\footnotetext{
"' Present address: INFIQC, Departamento de Físicoquímica, Universidad Nacional de Córdoba, Sucursal 16, C.C. 61. 5016, Córdoba, Argentina.
}

transferred per collision in collisions of various collider gases with several excited molecules: azulene, ${ }^{2,3}$ 112-triffuoroethane, ${ }^{4}$ benzene, ${ }^{5}$ and toluene. ${ }^{6}$ The IRF technique has also been used to investigate the mechanisms and importance of $V-V$ energy transfer between several excited benzene derivatives and $\mathrm{CO}_{2}{ }^{7,8}$ The time dependent thermal lensing technique (TDTL) has been used to study energy transfer from $\mathrm{NO}_{2}$ to the rare gases 9 and experiments using excited $\mathrm{CS}_{2}$ are currently underway in this laboratory. ${ }^{10}$ Deactivation by the rare gases is an example of vibration to translation/rotation $(V-T / R)$ energy transfer (the rotations and translations are tightly coupled, and it is not possible to distinguish them in these particular experiments). Troe and co-workers have used the ultraviolet absorption technique (UVA) to follow the collisional deactivation of several molecules, ${ }^{11}$ including azulene, ${ }^{12}$ some benzene derivatives, ${ }^{13} \mathrm{CS}_{2},{ }^{14}$ and $\mathrm{SO}_{2} \cdot{ }^{15}$ They have also used a multiphoton photoexcitation method in competition with a chemical isomerization reaction to obtain information about energy transfer at very high excitation energies. ${ }^{16}$ Weston and Flynn and their coworkers $^{17-19}$ have used the infrared tunable diode laser (TDL) absorption technique to study energy transfer in several systems.

These experimental measurements have generally confirmed the ordering of relative collision efficiencies earlier found in unimolecular reaction studies. ${ }^{20}$ In addition, it has been found that the average energy transferred in a collision depends on the energy of the excited molecule. This dependence is nearly linear at low energies, but may "roll off" and become nearly constant at higher energies for some mole- 
cules investigated. Classical trajectory calculations and statistical-dynamical models ${ }^{21-23}$ have been applied to some of these systems and they have shown both successes and failures at a semiquantitative level. In particular, the classical trajectory calculations carried out by Lim and Gilbert ${ }^{24}$ are successful in describing collisional deactivation of azulene by the heavy monatomic gases, but they show poor agreement with experiment for the light monatomic gases. The discrepancies for the lighter colliders may be due to inaccuracies in the empirical potential energy surfaces employed and to various quantum effects, which are neglected in the quasiclassical calculations. One particular dynamical quantum effect ${ }^{25}$ could be ruled out on the basis of experiments ${ }^{26}$ using ${ }^{3} \mathrm{He}$ and ${ }^{4} \mathrm{He}$ collider gases, but other quantum effects are possible, including a fundamental difference between classical and quantum statistics. ${ }^{26}$ At this time, the validity of classical trajectory calculations as applied to large molecule energy transfer remains in doubt.

Deuteration furnishes an indirect means of testing for quantum effects, because the high frequencies of the vibrational modes involving hydrogen motions are reduced and become more classical. In the present work, we have extended the infrared fluorescence (IRF) measurements previously carried out with normal benzene $e^{5}$ and toluene ${ }^{6}$ to the corresponding perdeutero compounds. Gas phase toluene- $d_{8}$ and benzene- $d_{6}$ are excited to the $S_{1}$ state, which subsequently undergoes radiationless transitions to form highly excited molecules in the electronic ground state. Infrared emission is then monitored as a function of time to determine the energy-transfer parameters.

Deuteration furnishes only an indirect test for quantum effects, because large molecules with high levels of excitation in the quasicontinuum provide a complicated energy-transfer system where $V-V$ and $V-T / R$ can take place simultaneously, when the collision partner is larger than a monatomic. This situation is quite unlike the case for small molecules at low levels of excitation, where resonant energy transfer takes place. ${ }^{27}$ It is our aim that the present work will contribute to the growing body of high quality energy transfer data which can be used for testing the validity of theories of large molecule energy transfer.

\section{BENZENE- $d_{6}$ AND TOLUENE- $d_{8}$ PHOTOPHYSICS}

The fundamental requirements of the IRF technique are that (a) the extent of unimolecular reaction must be small (see below), and (b) the rate of internal conversion (IC) or intersystem crossing (ISC) to the ground state $\left(S_{0}\right)$ in the excited molecules must be rapid compared to collisional deactivation and infrared fluorescence in the excited electronic state $\left(S_{1}\right)$. Therefore, it is important to know the rates of the radiationless processes and the amount of energy that is lost by fluorescence from the excited $S_{1}$ state. For a full understanding, the effects of deuterium substitution on the rates of IC, ISC, and fluorescence must be known. Unfortunately, this knowledge is not complete for some of the molecules investigated in the present work, but uscful guidance is obtained from other molecular species.

The major effect of deuterium substitution on the triplet excited state appears to be a reduction, due to the Franck-
Condon effect, of the rate constant for ISC to the ground state, $T_{1} \rightarrow S_{0}$ : for instance, the phosphorescence lifetimes of $\mathrm{C}_{6} \mathrm{H}_{6}$ and $\mathrm{C}_{6} \mathrm{D}_{6}$ are 7 and $28 \mathrm{~s}$, respectively. ${ }^{28}$ The FranckCondon factor depends on the vibrational wave function overlap between the lowest vibrational level of $T_{1}$ and an isoenergetic high vibrational level of $S_{0}$. For degenerate vibrational levels in the two states, this overlap decreases as the quantum number increases. For a given energy gap, the high frequency $\mathrm{C}-\mathrm{H}$ stretching vibrations are dominant in accepting the vibrational energy in a radiationless transition, because fewer quanta of high-frequency $\mathrm{C}-\mathrm{H}$ modes are required than for modes which involve $\mathrm{C}-\mathrm{D}$ stretches. As a consequence, the deuterated molecule has a slower rate of radiationless decay to the ground state. ${ }^{29}$

However, the situation is different at higher energy, because the decay rate for the deuterated molecule increases more rapidly with energy than does that of the normal species, perhaps because the vibrational density of states increases faster in the deuterated species. In $\mathrm{C}_{6} \mathrm{H}_{6}$, the $\mathrm{C}-\mathrm{H}$ modes are thought to be the dominant accepting modes, with little contribution from the lower frequency modes. In benzene- $d_{6}$, the $\mathrm{C}-\mathrm{D}$ modes are less efficient as accepting modes (because they are lower in frequency than the $\mathrm{C}-\mathrm{H}$ modes) and other modes can also contribute significantly as acceptors, but the overall efficiency is still low. The advantage of the $\mathrm{C}_{6} \mathrm{H}_{6}$ high frequency modes at the $T_{1}$ energy origin decreases with increasing energy so that the rates for $\mathrm{C}_{6} \mathrm{H}_{6}$ and $\mathrm{C}_{6} \mathrm{D}_{6}$ approach one another at high energy. In the particular case of toluene, deuteration has only a minor effect: the phosphorescence lifetime is $8 \mathrm{~s}$ for toluene- $d_{0}$ and $10.5 \mathrm{~s}$ for toluene- $d_{8} .{ }^{30}$

The deuterium isotope effect on singlet state lifetimes has not been studied as extensively as that for the triplet states. At the $S_{1}$ origin, the IC rates are $0.54 \times 10^{-6} \mathrm{~s}^{-1}$ for $\mathrm{C}_{6} \mathrm{D}_{6}$ and $1.6 \times 10^{-6} \mathrm{~s}^{-1}$ for $\mathrm{C}_{6} \mathrm{H}_{6} \cdot{ }^{28}$ But the IC rate increases more rapidly as a function of excitation energy for the deuterated molecule, as is observed for $T_{1} \rightarrow S_{0}$ ISC process discussed above.

In the present experiments, no effects were observed that were directly attributable to increased lifetimes of the excited electronic states due to deuteration. It appears that although deuteration lowers the radiationless rates, they are still sufficiently rapid at the initial excitation energy $\left(\sim 41000 \mathrm{~cm}^{-1}\right)$ so that most of the molecules are found in highly vibrationally excited $S_{0}$ states after $\sim 1 \mu \mathrm{s}$, as is observed for the normal molecules.

\section{EXPERIMENT}

The collisional relaxation of toluene- $d_{8}$ and benzene- $d_{6}$ was monitored with the IRF technique, which has been described elsewhere. ${ }^{5,6}$ Basically, a KrF excimer laser (248 $\mathrm{nm}$ ) irradiated the gas phase species in a $30 \mathrm{~cm}$ long, $4.5 \mathrm{~cm}$ diameter Pyrex cell. IRF near $4.3 \mu \mathrm{m}$ from the C-D stretching modes was viewed through $\mathrm{a} \mathrm{CaF}_{2}$ side window with a 3 mm diameter $77 \mathrm{~K} \mathrm{InSb}$ photovoltaic detector (Infrared Associates) equipped with a matched preamplifier and an appropriate interference filter. The signals were amplified with a Tektronix AM 502 ac-coupled amplifier and averaged with 
a LeCroy 9400 digital oscilloscope for $\sim 5000$ pulses, in order to achieve good $S / N$ ratios in each experiment. The signal was further analyzed after transfer to a Macintosh computer. The IRF signal risetime was limited by the $\sim 5 \mu$ s rise time of the infrared detector/preamplifier.

The infrared interference filter used in the experiments was centered at $4.4 \mu \mathrm{m}(0.68 \mu \mathrm{m}$ FWHM $)$ and did not transmit all of the emission from the methyl C-D stretches. To determine whether this affected the shapes of the decay curves, experiments were also performed using a broadband filter $(\sim 3-5 \mu \mathrm{m})$ and the results were compared. The comparison showed that the truncation of the emission did not significantly affect the decay curves and thus does not significantly affect the derived energy transfer parameters.

The average laser fluence employed in the present measurements was $\sim 25 \mathrm{~mJ} \mathrm{~cm}^{-2}$, so that $\sim 0.5 \%$ of the molecules in the laser beam were excited. Laser beam transmittance measurements at $248 \mathrm{~nm}$ gave an absorption cross section (base $e$ ) of $(3.6 \pm 0.2) \times 10^{-19} \mathrm{~cm}^{2}$ for toluene- $d_{8}$ and $(3.5 \pm 0.2) \times 10^{-19} \mathrm{~cm}^{2}$ for benzene- $d_{6}$ (Beer's Law was assumed). These values are very similar to the corresponding cross sections measured in this laboratory for toluene- $d_{0}$ (Ref. 6) and benzene- $d_{0} .{ }^{5}$

The "parent" gases, toluene- $d_{8}$ (Sigma, $99+$ at \% D) and benzene- $d_{6}$ (Aldrich, $99.5+$ at $\% \mathrm{D}$ ), were degassed prior to use. Flowing conditions were employed for pure toluene- $d_{8}$ and benzene- $d_{6}$ decay measurements with pressures in the cell varying from 10 to 50 mTorr. Collider gas experiments were performed under static bulb conditions in mixtures containing $15 \mathrm{~m}$ Torr of parent gas and up to about $800 \mathrm{mT}$ Torr of collider. The collider gases were all research grade and were used without further purification. The gas samples were introduced into the cell through a fine-control needle valve and pressures were monitored with a $0-1$ Torr capacitance manometer (MKS Baratron model 227). A1though a slow decrease in the pressure of pure parent was observed while irradiating under static bulb conditions, these effects were small. Some polymeric deposition on the walls has been reported in the literature for higher pressure conditions; in our experiments (involving much lower pressures) a brown film developed on the windows at the sites of entry and exit of the laser beam and a hazy film of material on the Pyrex cell walls became apparent only after many experimental runs were completed. In order to determine the extent of parent photodecomposition in the static cell experiments, we used a modulated molecular beam mass spectrometer (MMB-MS) detection technique ${ }^{5}$ to search for photoproducts. The quantum yield for parent loss was found to be $3.5 \% \pm 1.5 \%$, and the only gaseous product detected was $\mathrm{D}_{2}$.

\section{RESULTS}

The experimental results consist of IRF decay curves, which were fitted by nonlinear least squares to an empirical function,

$$
\langle\langle I(t)\rangle\rangle=A \exp \left(-k^{I} t+b^{\prime} t^{2}\right)+B .
$$

The double brackets indicate that the observed IRF corresponds to the bulk average over the population distribu- tion, which evolves with time. The theoretical relationship between IRF emission intensity and vibrational energy $(E)$ has been discussed elsewhere, ${ }^{2,3}$ and it was used with known vibrational assignments ${ }^{31}$ for the excited molecules to calculate $I(E)$ "calibration functions." These functions depend on densities of states ratios and are not very sensitive to the details of the vibrational assignments. For convenience, the calibration functions used in the present work are collected in Table I. These functions were used to invert (hence, the subscript "inv") the IRF data to give the bulk average energy $\langle\langle E(t)\rangle\rangle_{\text {inv }}$ and the bulk average energy transferred per collision $\langle\langle\Delta E\rangle\rangle_{\mathrm{inv}}$, using the procedure described elsewhere. ${ }^{6}$ The results of this analysis are plots of $\langle\langle\Delta E\rangle\rangle_{\text {inv }}$ as a function of $\langle\langle E\rangle\rangle_{\text {inv }}$ for each experimental run, which is characterized by a mixing ratio of parent/collider. Note that in the experiments the quantity measured is the absolute rate of energy loss, which is proportional to the product $k_{\mathrm{LJ}} \cdot\langle\langle\Delta E\rangle\rangle_{\text {inv }}$, where $k_{\mathrm{LJ}}$ is the bimolecular collision rate constant calculated ${ }^{20.21}$ from the Lennard-Jones parameters (Table II).

Each mixture of parent and collider is characterized by a "collision fraction," which is defined as the fraction of collisions due to parent-collider gas interactions,

$$
\text { Collision Fraction }=\frac{[\mathrm{C}] k_{\mathrm{LJ}}^{c}}{[\mathrm{C}] k_{\mathrm{LJ}}^{c}+[\mathrm{P}] k_{\mathrm{LJ}}^{P}},
$$

where $[\mathrm{C}]$ and $[\mathrm{P}]$ denote concentrations of collider gas and parent, respectively. An example of a plot of $\langle\langle\Delta E\rangle\rangle_{\mathrm{inv}}$ vs collision fraction is shown in Ref. 26 , where a slight nonlinearity is observed. The average total amounts of energy transferred per collision were extrapolated to unit collision fraction in order to obtain $\langle\langle\Delta E\rangle\rangle_{\text {inv }}$ for parent-collider gas collisions, uncontaminated by the effects of parent-parent gas collisions. ${ }^{6}$ The extrapolated $\langle\langle\Delta E\rangle\rangle_{\text {inv }}$ for a few of the systems studied are shown in Figs. 1 and 2, and in Table II are presented $\langle\langle\Delta E\rangle\rangle_{\mathrm{inv}}$ values at $\langle\langle E\rangle\rangle_{\mathrm{inv}}=24000 \mathrm{~cm}^{-1}$ (this energy is convenient for comparisons with results obtained for azulene ${ }^{2,3}$ ). The uncertainties given in the table and shown in the figures were estimated by propagation of statistical errors; possible systematic errors were not included. Note in Fig. 1, that the values for the normal isotopomers of benzene and toluene show virtually identical behavior and deuteration causes both a decrease in the magnitudes of $\langle\langle\Delta E\rangle\rangle_{\text {inv }}$ and a change in the energy dependence.

Also presented in Table II are polynomial coefficients approximately describing the microcanonical collision step size for down steps, $\langle\Delta E\rangle_{d}$, based on the exponential modcl

TABLE I. IRF calibration functions: $\ln (E)=\Sigma_{k=0}^{4} a_{k}(\ln I)^{k}$.

\begin{tabular}{lccccc}
\hline \hline & $a_{0}$ & $a_{1}$ & $a_{2}$ & $10^{3} a_{3}$ & $10^{5} a_{4}$ \\
\hline $\begin{array}{l}\text { Benzene- } d_{0} \\
\left(3073 \mathrm{~cm}^{-1}\right)\end{array}$ & 11.6487 & 0.871 & 0.0767 & 2.93 & $\cdots$ \\
$\begin{array}{l}\text { Benzene- } d_{\mathrm{o}} \\
\left(2287 \mathrm{~cm}^{-1}\right)\end{array}$ & 11.3351 & 0.871 & 0.0678 & 1.62 & -2.55 \\
$\begin{array}{l}\text { Toluene- } d_{0} \\
\left(3056 \mathrm{~cm}^{-1}\right)\end{array}$ & 11.9241 & 0.897 & 0.0932 & 6.40 & +22.6 \\
$\begin{array}{l}\text { Toluene- } d_{8} \\
\left(2286 \mathrm{~cm}^{-1}\right)\end{array}$ & 11.5804 & 0.853 & 0.0584 & -1.07 & -31.5 \\
\hline \hline
\end{tabular}


TABLE II. Collider gas data for the deactivation of toluene- $d_{8}$, benzene- $d_{6}$, and benzene- $d_{0}$.

\begin{tabular}{|c|c|c|c|c|c|c|c|}
\hline Collider & $\begin{array}{l}\sigma_{\mathrm{L}}{ }^{2} \\
(\AA)\end{array}$ & $\begin{array}{c}\epsilon / K^{2} \\
(\mathrm{~K})\end{array}$ & $\begin{array}{c}10^{10} k_{\mathrm{LJ}} \\
\left(\mathrm{cm}^{3} \mathrm{~s}^{-1}\right)\end{array}$ & $\begin{array}{c}-\langle\langle\Delta E\rangle\rangle_{\mathrm{inv}}^{\mathrm{b}, \mathrm{c}} \\
\left(\mathrm{cm}^{-1}\right)\end{array}$ & $\begin{array}{c}C_{1} \\
\left(\mathrm{~cm}^{-1}\right)\end{array}$ & $\begin{array}{c}\langle\Delta E\rangle_{d}{ }^{\mathbf{d}} \\
C_{2}\end{array}$ & $\begin{array}{c}10^{7} C_{3} \\
\left(1 / \mathrm{cm}^{-1}\right)\end{array}$ \\
\hline \multicolumn{8}{|l|}{ Toluene- $d_{8}$} \\
\hline$C_{7} D_{3}$ & 5.92 & 410 & 7.02 & $747 \pm 13$ & 30.5 & 0.0611 & -9.48 \\
\hline${ }^{3} \mathrm{He}^{e}$ & 2.55 & 10 & 8.20 & $53 \pm 2$ & 41.8 & 0.0061 & -0.74 \\
\hline${ }^{4} \mathrm{He}$ & 2.55 & 10 & 7.13 & $60 \pm 1$ & 37.4 & 0.0080 & -1.25 \\
\hline $\mathrm{Ne}$ & 2.82 & 32 & 4.23 & $87 \pm 3$ & 46.3 & 0.0103 & -1.66 \\
\hline $\mathrm{Ar}$ & 3.47 & 114 & 4.48 & $126 \pm 3$ & 54.1 & 0.0135 & -2.17 \\
\hline $\mathrm{Kr}$ & 3.66 & 178 & 4.07 & $126 \pm 4$ & 49.6 & 0.0129 & -1.88 \\
\hline $\mathrm{Xe}$ & 4.05 & 230 & 4.15 & $138 \pm 5$ & 55.1 & 0.0129 & -1.68 \\
\hline $\mathbf{H}_{2}$ & 2.83 & 60 & 13.56 & $82 \pm 3$ & 43.2 & 0.0092 & -1.26 \\
\hline$D_{2}$ & 2.73 & 69 & 9.67 & $81 \pm 3$ & 39.2 & 0.0091 & -1.19 \\
\hline $\mathrm{N}_{2}$ & 3.74 & 82 & 5.22 & $121 \pm 4$ & 32.1 & 0.0111 & -0.97 \\
\hline $\mathrm{O}_{2}$ & 3.48 & 103 & 4.88 & $173 \pm 11$ & 114.8 & 0.0122 & -1.62 \\
\hline $\mathrm{CO}$ & 3.70 & 105 & 5.39 & $133 \pm 5$ & 32.0 & 0.0130 & -1.49 \\
\hline $\mathrm{H}_{2} \mathrm{O}$ & 2.71 & 506 & 7.08 & $387 \pm 14$ & 56.3 & 0.0237 & -1.18 \\
\hline $\mathrm{D}_{2} \mathrm{O}$ & 2.71 & 506 & 6.77 & $378 \pm 25$ & 35.5 & 0.0225 & -0.49 \\
\hline $\mathrm{CH}_{4}$ & 3.79 & 153 & 7.38 & $199 \pm 6$ & -55.6 & 0.0179 & -2.47 \\
\hline $\mathrm{NH}_{3}$ & 2.90 & 558 & 7.75 & $350 \pm 12$ & -1.7 & 0.0327 & -4.86 \\
\hline $\mathrm{SF}_{6}$ & 5.20 & 212 & 4.96 & $337 \pm 12$ & 46.4 & 0.0246 & -2.28 \\
\hline$c-\mathrm{C}_{3} \mathrm{H}_{6}$ & 4.63 & 299 & 6.78 & $461 \pm 18$ & 46.0 & 0.0281 & -1.51 \\
\hline $\mathrm{C}_{3} \mathrm{H}_{6}$ & 4.78 & 271 & 6.83 & $441 \pm 15$ & 86.4 & 0.0317 & -3.90 \\
\hline$n-\mathrm{C}_{4} \mathrm{H}_{10}$ & 5.40 & 307 & 7.04 & $530 \pm 20$ & -3.3 & 0.0394 & -4.18 \\
\hline \multicolumn{8}{|l|}{ Benzene- $d_{6}$} \\
\hline$C_{6} D_{6}$ & 5.27 & 440 & 6.27 & $576 \pm 6$ & 61.3 & 0.0452 & -6.60 \\
\hline $\mathrm{H}_{2}$ & 2.83 & 60 & 11.80 & $61 \pm 1$ & 38.0 & 0.0071 & -0.92 \\
\hline $\mathrm{D}_{2}$ & 2.73 & 69 & 8.43 & $46 \pm 1$ & 28.6 & 0.0055 & -0.53 \\
\hline \multicolumn{8}{|l|}{ Benzene- $d_{0}{ }^{f}$} \\
\hline $\mathrm{C}_{6} \mathrm{H}_{6}$ & 5.27 & 440 & 6.5 & $869 \pm 17$ & 41.5 & 0.0539 & -4.58 \\
\hline${ }^{3} \mathrm{He}^{\mathrm{e}}$ & 2.551 & 10.22 & 5.37 & $26 \pm 1$ & 20.1 & 0.00367 & -0.019 \\
\hline${ }^{4} \mathrm{He}$ & 2.551 & 10.22 & 6.16 & $23 \pm 1$ & 20.7 & 0.00421 & -0.453 \\
\hline $\mathrm{Ne}$ & 2.82 & 32.8 & 3.75 & $20 \pm 1$ & 18.7 & 0.00442 & -0.777 \\
\hline Ar & 3.542 & 93.3 & 4.07 & $30 \pm 3$ & 17.8 & 0.00582 & -0.991 \\
\hline $\mathrm{Kr}$ & 3.655 & 178.9 & 3.8 & $34 \pm 1$ & 28.4 & 0.00521 & -0.738 \\
\hline $\mathrm{Xe}$ & 4.047 & 231 & 3.95 & $36 \pm 2$ & 28.0 & 0.00594 & -0.986 \\
\hline $\mathrm{H}_{2}$ & 2.827 & 59.7 & 11.77 & $66 \pm 3$ & 34.2 & 0.00779 & -0.982 \\
\hline $\mathrm{D}_{2}$ & 2.73 & 69 & 8.42 & $45 \pm 2$ & 29.8 & 0.00716 & -1.27 \\
\hline $\mathrm{N}_{2}$ & 3.798 & 71.4 & 4.67 & $34 \pm 2$ & 27.6 & 0.00526 & -0.778 \\
\hline $\mathrm{O}_{2}$ & 3.467 & 106.7 & 4.42 & $37 \pm 2$ & 19.5 & 0.00625 & -0.938 \\
\hline $\mathrm{CO}$ & 3.69 & 91.7 & 4.75 & $37 \pm 4$ & 25.9 & 0.00591 & -0.902 \\
\hline $\mathrm{CO}_{2}$ & 3.941 & 195.2 & 4.93 & $208 \pm 4$ & 47.7 & 0.0202 & -3.11 \\
\hline $\mathrm{H}_{2} \mathrm{O}$ & 2.641 & 809.1 & 6.94 & $373 \pm 36$ & 84.4 & 0.0277 & -3.51 \\
\hline $\mathrm{NH}_{3}$ & 2.9 & 558.3 & 6.9 & $348 \pm 78$ & 2.85 & 0.0232 & -0.957 \\
\hline $\mathrm{CH}_{4}$ & 3.758 & 148.6 & 6.54 & $163 \pm 17$ & 15.1 & 0.0173 & -2.34 \\
\hline $\mathrm{SF}_{6}$ & 5.128 & 222.1 & 4.79 & $538 \pm 49$ & 48.2 & 0.0398 & -0.482 \\
\hline$c-\mathrm{C}_{3} \mathrm{H}_{6}$ & 4.807 & 248.9 & 6.28 & $635 \pm 120$ & 27.3 & 0.0616 & -12.05 \\
\hline $\mathrm{C}_{3} \mathrm{H}_{6}$ & 4.78 & 271 & 6.36 & $424 \pm 56$ & $31.9^{-}$ & 0.0418 & -7.57 \\
\hline$n-\mathrm{C}_{4} \mathrm{H}_{10}$ & 4.687 & 531.4 & 6.56 & $615 \pm 50$ & 32.9 & 0.0535 & -9.044 \\
\hline
\end{tabular}

Lennard-Jones parameters (for collider gases) from Refs. 11 and 12 . When parameters for deuterated species are not available, the parameters for the corresponding normal isotopomer are used.

${ }^{b}$ Uncertainties are $\pm 2 \sigma$ statistical errors; possible systematic errors are not included.

${ }^{\mathrm{c}}$ Evaluated at $\langle\langle E\rangle\rangle_{\mathrm{inv}}=24000 \mathrm{~cm}^{-1}$.

${ }^{\mathrm{d}}\langle\Delta E(E)\rangle_{d}=C_{1}+C_{2} E+C_{3} E^{2}$.

'Reanalyzed data from Ref. 26.

'Reanalyzed data from Ref. 5.

for the collision step size distribution. These polynomial coefficients were obtained by least squares fitting of the data to the following approximate form, as described elsewhere: ${ }^{6}$

$$
\langle\langle\Delta E\rangle\rangle_{\mathrm{inv}} \approx D^{-1}-\langle\Delta E\rangle_{d},
$$

$$
D=\langle\Delta E\rangle_{d}^{-1}+(k T)^{-1}-B .
$$

The factor $B=d\{\ln [\rho(E)]\} / d E$ is based on the WhittenRabinovitch approximation for the density of rovibrational states, ${ }^{32,33}$ 


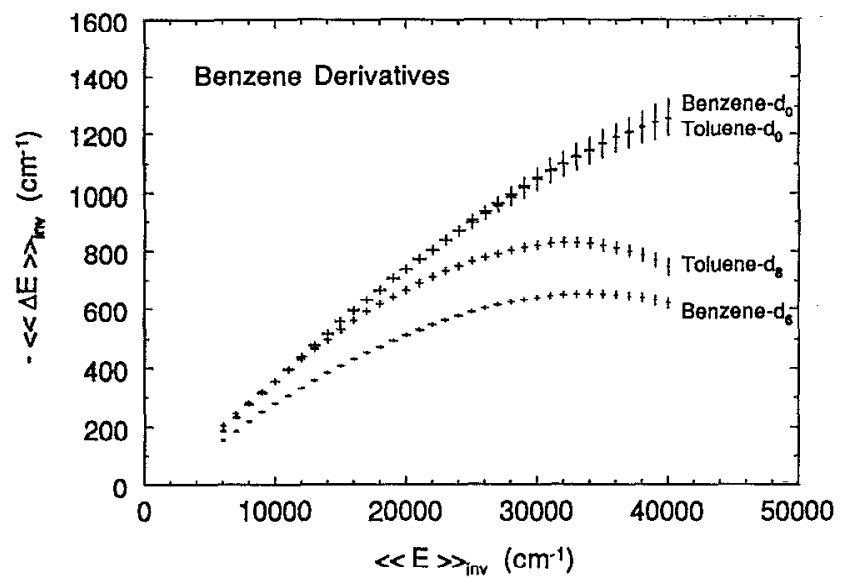

FIG. 1. Average energy transferred per collision as a function of the average vibrational energy for isotopically substituted benzene derivatives deactivated in collisions with the unexcited species. The error bars are $\pm 2 \sigma$ statistical errors (see Refs. 5 and 6 for an explanation).

$$
B=\frac{s-1+r / 2}{E+a(E) E_{z}}
$$

where $s$ is the number of vibrations, $r$ is the number of rotations, $E_{\mathrm{z}}$ is the zero point energy, and $a(E)$ is the WhittenRabinovitch parameter. ${ }^{32,33}$ For present purposes, the rovibrational energy $E$ is identified with $\langle\langle E\rangle\rangle_{\text {inv }}$ and the least squares fitting is carried out by expanding $\langle\Delta E(E)\rangle_{d}$ as a
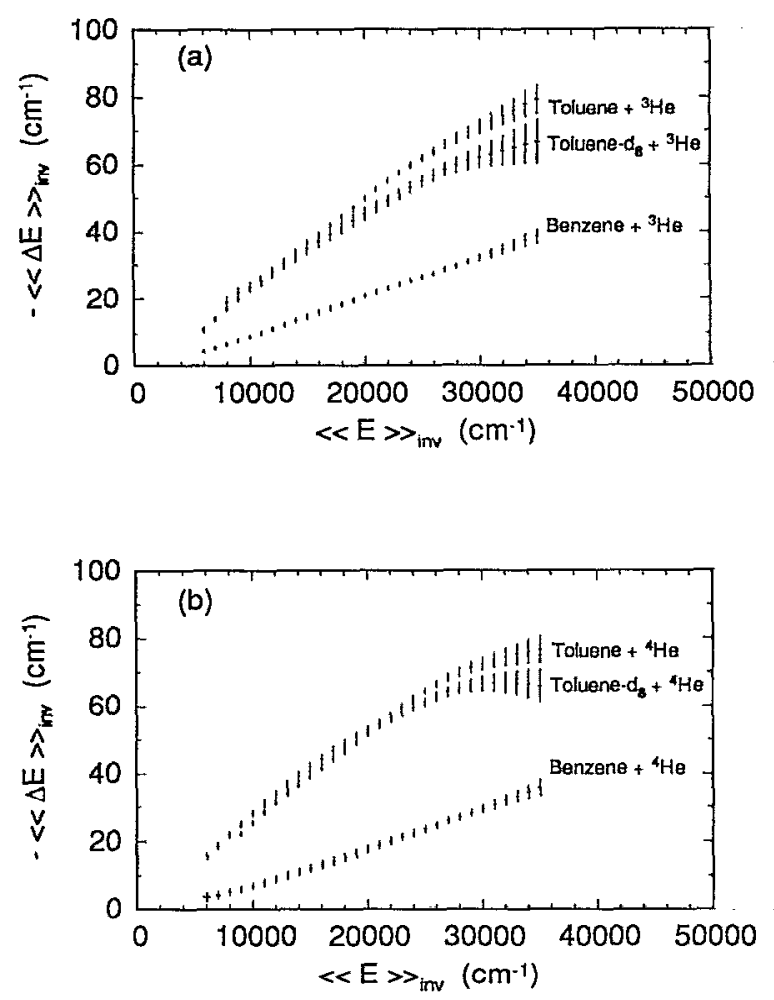

FIG. 2. Average energy transferred per collision as a function of the average vibrational energy for isotopically substituted benzene derivatives deactivated in collisions with ${ }^{3} \mathrm{He}$ and ${ }^{4} \mathrm{He}$. polynomial, and fitting the coefficients. A second order polynomial fits the data satisfactorily: $\langle\Delta E(E)\rangle_{d}$ $=C_{1}+C_{2} E+C_{3} E^{2}$.

These equations can be used with the Whitten-Rabinovitch parameters summarized in Table III to obtain numerical values for the experimental $\langle\langle\Delta E\rangle\rangle_{\mathrm{inv}}$ vs $\langle\langle E\rangle\rangle_{\mathrm{inv}}$ curves for all of the collision pairs (most of which are not shown in the figures). Moreover, $\langle\Delta E(E)\rangle_{d}$ can be used directly in master equation calculations.

The experinental results obtained previously ${ }^{5}$ for benzene- $d_{0}$ were not analyzed by the method described above and the use of different analysis methods can lead to slightly different quantitative results. Thus the experimental data for benzene- $d_{0}$ obtained previously were reanalyzed according to the present procedure and the revised results are presented in Table II.

The results summarized in Table II for an average vibrational energy of $24000 \mathrm{~cm}^{-1}$ show that the $\langle\langle\Delta E\rangle\rangle_{\text {inv }}$ values for toluene- $d_{8}$ are within $\sim 20 \%$ of those for toluene- $d_{0}$ (Ref. 6). This result is in reasonable agreement with unimolecular reaction studies involving deuterated species, where little effect due to deuteration is seen. ${ }^{34,35}$ For the benzene isotopomers, the differences due to deuteration are somewhat larger than for toluene, but the differences between benzene and toluene for all collider gases except the parent are much larger than the differences introduced by deuteration. Also, deuterium substitution in the collider gases sometimes produces much larger variations than those produced by deuteration of the excited species, as shown in Fig. 3.

The results at $24000 \mathrm{~cm}^{-1}$ do not tell the whole story, however. Deuteration of the excited molecule appears to change the energy dependence of $\langle\langle\Delta E\rangle\rangle_{\text {inv }}$ and $\langle\Delta E\rangle_{d}$, as shown in Fig. 1, where the excited parent species are deactivated by the corresponding unexcited species. Note that the plots of $\langle\langle\Delta E\rangle\rangle_{\text {inv }}$ vs $\langle\langle E\rangle\rangle_{\text {inv }}$ for the deuterated species show that $\langle\langle\Delta E\rangle\rangle_{\text {inv }}$ is somewhat smaller than for the normal species and $\langle\langle\Delta E\rangle\rangle_{\text {inv }}$ actually exhibits a maximum and then decreases as the vibrational energy is increased further. This effect is especially striking for toluene- $d_{8}$. The fitted coefficients for $\langle\Delta E\rangle_{d}$ in Table II reflect this effect and although deuteration seems to change the magnitudes of the coefficients, the signs are unchanged, possibly indicating that $\langle\langle\Delta E\rangle\rangle_{\text {inv }}$ for the normal species exhibits the same sort of maximum, but it lies beyond the range of the experimental data. Troe and co-workers have reported ${ }^{1,11}$ that $\langle\langle\Delta E\rangle\rangle$ becomes independent of energy and the energy dependence

TABLE III. Whitten-Rabinovitch parameters. ${ }^{\text {" }}$

\begin{tabular}{llccc}
\hline \hline & $\begin{array}{c}\text { Zero point } \\
\text { energy } \\
\left(\mathrm{cm}^{-1}\right)\end{array}$ & $\beta$ & $\begin{array}{c}\text { Vibrational } \\
\text { degrees of } \\
\text { freedom }(\mathrm{s})\end{array}$ & $\begin{array}{c}\text { Rotor degrees } \\
\text { of freedom }(r)\end{array}$ \\
\hline Benzene- $d_{0}$ & 21399.35 & 1.3281 & 30 & 0 \\
Benzene- $d_{0}$ & 17290.5 & 1.2616 & 30 & 0 \\
Toluene- $d_{0}$ & 27109.0 & 1.3644 & 38 & 1 \\
Toluene- $d_{8}$ & 21708 & 1.3010 & 38 & 1 \\
\hline \hline
\end{tabular}

"Defined in Ref. 32. 


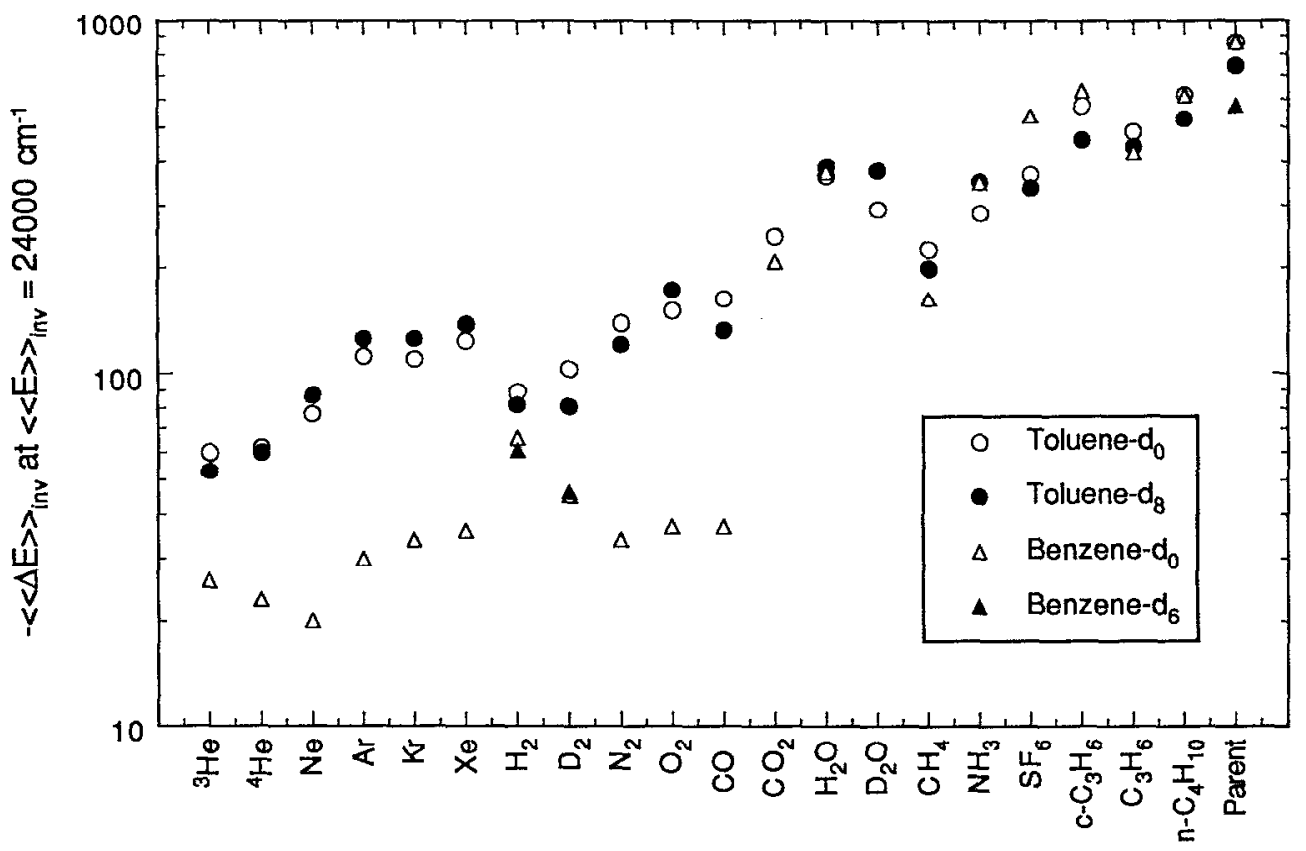

FIG. 3. A comparison of the average energy transferred per collision (at $\langle E\rangle_{\mathrm{inv}}=24000 \mathrm{~cm}^{-1}$ ) in deactivating benzene and toluene isotopomers.

exhibited here by the deuterated species may be another manifestation of the same behavior.

It is also possible that the change in energy dependence on deuteration is an artifact caused by (1) possible lengthening of the excited electronic state lifetimes, or (2) the different transmission bandwidths of the infrared filters used in the expcriments may result in different amounts of the underlying IRF emission continuum ${ }^{36}$ being observed. As mentioned above, no direct indications of effects due to increased lifetimes were observed, but we cannot rule out relatively subtle effects, such as the change in energy dependence. The infrared continuum is observed in highly excited benzene- $d_{0}$ and probably it originates from combination and difference bands that have some $\mathrm{C}-\mathrm{H}$ stretch character. The results obtained in other experiments ${ }^{36}$ indicate that the continuum emission decays more rapidly than the $\mathrm{C}-\mathrm{H}$ stretch fundamental band and it contributes $5 \%-10 \%$ to the total band intensity at the initial excitation cnergy. Bccause the difference and combination bands are not included in the theoretical calculation of IRF emission intensity, the presence of the continuum can affect $\langle\langle\Delta E\rangle\rangle_{\mathrm{inv}}$. The magnitude of this possible effect in the present experiments is difficult to estimate, because the emission spectra of excited benzene- $d_{6}$ and the excited toluene isotopomers have not yet been obtained. However, the fact that there is no significant difference between results obtained with a narrow band filter and a broadband filter (see above) would indicate that contributions from the continuum do not significantly affect the results. The effect of the continuum will be further investigated in future experiments.

\section{DISCUSSION}

In a recent paper, ${ }^{8}$ we hypothesized that the collisional deactivation of large molecules can be described with the following components: (1) $V-T / R$ energy transfer takes place with all colliders, probably due to the short range repulsive interactions invoked in the biased random walk mod$\mathrm{el}^{22,24}$ and found to be important in trajectory calculations on smaller molecules; ${ }^{23}$ (2) $V-V$ energy transfer can take place by long range dipole-dipole interactions, ${ }^{8}$ and thus resonance may play a role; ( 3 ) if the collider gas is polar, it is likely that the permanent dipole can interact with the vibrating dipoles of the excited molecule and therefore $V-R$ energy transfer will be enhanced; (4) if low-lying excited electronic states are present, they may substantially enhance energy transfer rates. ${ }^{9}$ It is also possible that quantum effects play a role in each of the components listed above and the mass change due to isotopic substitution may make these quantum effects apparent. The present results tend to confirm and extend this view of energy transfer.

\section{Possible quantum effects}

Quantum effects were invoked by Gilbert and Zare ${ }^{25}$ as one possible explanation for the uneven performance of classical trajectory calculations ${ }^{23}$ in describing deactivation of highly vibrationally excited azulene by the rare gases. Trajectory calculations for deactivation by the heavier rare gases agreed well with experimental data, while those for the lighter rare gases showed large discrepancies with experiment. Two possible explanations were proposed: (1) the potential energy surfaces for the azulene + lighter rare gases are in error; ${ }^{23}$ and (2) quantum effects are important for the lighter systems, but not for the heavier ones. ${ }^{25}$ The proposed quantum effects were thought to arise from two possible sources: (1) zero point energy, which is conserved in quantum systems, but can be transferred in classical trajectory calculations; and (2) a dynamical quantum effect due to interferences among matter waves with small deffection an- 
gles at large impact parameters (these same interferences are responsible for the finite quantum total elastic cross sections, while the corresponding classical cross sections are infinite).

Gilbert and Zare proposed ${ }^{25}$ a test for the dynamical quantum effect: that deactivation of excited species by ${ }^{3} \mathrm{He}$ and ${ }^{4} \mathrm{He}$ be investigated. These colliders have a relatively large mass difference, but the potential energy surface is unaffected, and the average energy transferred should be affected significantly if the dynamical quantum effect is important. A preliminary publication from this laboratory ${ }^{26}$ reported experiments that showed the two helium isotopes produced nearly identical energy transfer parameters. The fully analyzed data, reported in Table II and in Fig. 2, confirm the preliminary report. The dynamical quantum effect is not important, if Gilbert and Zare are correct in their speculation that the ${ }^{3} \mathrm{He} /{ }^{4} \mathrm{He}$ mass difference will produce a large difference in energy transfer parameters.

However, quantum effects may still be important in defining the differences between quantum systems and classical trajectory calculations on energy transfer: quantum and classical population statistics are significantly different. ${ }^{26}$ In a classical system of coupled oscillators, the energy is randomized so that the average energy in each vibration mode is equal: equipartition of energy. In a quantum system, however, this is not the casc. In microcanonical quantum systems, the average energy in the $i$ th vibrational mode is given by the probability of finding $v_{i}$ quanta in the mode, multiplied by the energy in the mode, and then summed over all quantum levels of the mode. For coupled quantum systems which are described well in terms of densities of states, the average energy in a mode is given by

$$
\langle E\rangle_{i}=\frac{h v_{i}}{\rho_{s}(E)} \sum_{v_{1}=1}^{E / h v} v_{i} \rho_{s-1}\left(E-v_{i} h v_{i}\right)
$$

where $v_{t}$ is the mode vibrational frequency, $\rho_{s}(E)$ is the vibrational density of states at energy $E$ for $s$ modes, and $\rho_{s-1}(E)$ is the density of states for $s-1$ modes, omitting the $i$ th mode and the energy contained in it.

From Eq. (6), the high frequency modes in a quantum system are "energy poor," while the low frequency modes are "energy rich," as shown in Fig. 4. Classical equipartition of energy is not a good approximation, unless all of the modes contain a large amount of energy, relative to $h v_{i}$, or unless the vibrational frequencies are nearly degenerate. The zero point energy is not constrained in classical systems, and when it is included along with the disposable vibrational energy in classical trajectory calculations, the high frequency modes contain far too much vibrational energy. These effects combine to introduce a fundamental bias in classical trajectory calculations involving large molecules which possess a wide range of vibrational frequencies. For benzene- $d_{0}$ containing $\sim 10000 \mathrm{~cm}^{-1}$ of vibrational energy in addition to the zero point energy, the classically disposable average energy per mode is $\sim 1050 \mathrm{~cm}^{-1}$, which can be compared with the $\sim 58 \mathrm{~cm}^{-1}$ available in each $\mathrm{C}-\mathrm{H}$ stretching mode in the corresponding quantum system. This effect is familiar from the Whitten-Rabinovitch expressions for quantum state sums and densities, where the zero point energy ap-

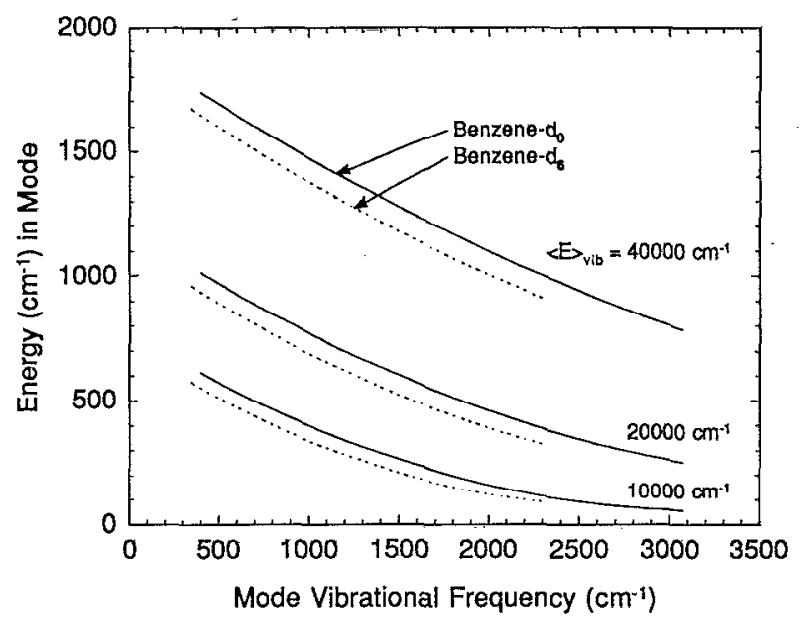

FIG. 4. Average disposable vibrational energy residing in the vibrational modes of benzene- $d_{0}$ and benzene- $d_{6}$ according to quantum statistics [Eq. (6) ], when the total disposable vibrational energy is 10000,20000 , and $40000 \mathrm{~cm}^{-1}$.

pears explicitly and the parameter $a(E)$ depends on the distribution of vibrational mode fundamental frequencies. If these factors are neglected, the resulting classical expression provides a very poor approximation to the quantum sums and densities of states over reasonable ranges of energy. $20,21,32,33$

It has been argued ${ }^{21}$ that large molecules behave essentially classically, because of their high densities of states. In the limit of very high energies, this conclusion is correct, but most molecules studied at chemically accessible energies have not reached the high energy classical limit. It is the inadequacy of the classical limit in describing quantum systems at chemical energies that leads to the bias discussed above. Note that the bias is less severe at high total energy and for smaller molecules (with a similar distribution of mode frequencies), but the bias is always present and may contribute to the successes and failures of classical trajectory simulations.

We previously proposed an explanation for the success of the classical calculations for light colliders and the failure for heavy colliders. ${ }^{26}$ The explanation was based on a combination of two effects: the bias present in classical trajectory calculations and the relative efficiency of deactivating the higher frequency modes by impulsive collisions, compared with adiabatic collisions. Apparently, this explanation is not correct, however, because recent classical trajectory calculations $^{37}$ carried out for normal and deuterated isotopomers give similar results, in agreement with experiment. Deuteration reduces the frequencies of the higher frequency modes, causing collisions to be more impulsive. If our explanation were correct, deuteration would result in more efficient energy transfer, contrary to experiment. Thus it would appear that the most likely explanation for the uneven performance of the trajectory calculations is that the assumed potential functions require adjustment, as suggested by Lim and Gilbert, ${ }^{23}$ and the effects of the bias are more subtle. 


\section{$\boldsymbol{V}-\boldsymbol{T} / \boldsymbol{R}$ and $\boldsymbol{V}-\boldsymbol{V}$ energy transfer mechanisms}

The effects of deuteration are presented in Fig. 4, which compares the average energy in each mode of benzene- $d_{0}$ and benzene- $d_{6}$, based on quantum statistics [Eq. (6)]. Only the higher frequency modes are affected significantly by deuteration: the $\mathrm{C}-\mathrm{D}$ stretch mode frequencies are about $2^{-1 / 2}$ times as large as those for the $\mathbf{C}-\mathrm{H}$ stretches and the mode energy contents are increased slightly. The lower frequency modes are practically unaffected: their frequencies and their average energy contents are lowered only slightly by deuteration.

We conclude from Fig. 4 that if collisional energy transfer occurs mostly by $V-T / R$ transfer from the lowest frequency modes, as expected from SSH theory, ${ }^{38-40}$ there will be little effect due to deuteration as long as intramolecular vibrational redistribution of energy (IVR) is slow, relative to the duration of a collision. Most of the experimental data presented in Fig. 3 are consistent with this notion. The slight decrease in $\langle\langle\Delta E\rangle\rangle_{\text {inv }}$ for the dcuterated isotopomers relative to the corresponding normal species for most colliders may be due in part to the slight lowering of average energy content in the low frequency modes in the deuterated molecules at the same total energy. This does not explain the larger effects seen for deactivation by the parent gases, however, where "sticky collisions" and $V-V$ energy transfer may be more important.

In experiments using $\mathrm{CO}_{2}$ as the collider gas, ${ }^{8} \mathrm{~V}-\mathrm{V}$ energy transfer to $\mathrm{CO}_{2}(001)$ was observed experimentally and explained semiquantitatively with a simple dipole-dipole model. The parameters uscd in the model are the vibrational frequencies and dipole transition matrix elements (obtained from infrared spectra), the Lennard-Jones radius, and an adjustable parameter related to the rate of IVR. Because the dipole-dipole interactions are effective over long ranges, straight line trajectories were assumed. The simple model gave reasonable fits to the experimental data on $\mathrm{CO}_{2}$ when reasonable IVR rates were assumed, and the calculated $V-V$ energy transfer was significant (although small), mostly because of the great strength of the $\mathrm{CO}_{2}$ transition matrix elements. In the present experiments, the dipole transition matrix elements are much weaker, even though the vibrational frequencies of excited parent and collider match exactly (in benzene* + benzene collisions, for example), and the simple model indicates that $V-V$ energy transfer is insignificant, if the IVR rates that fitted the $\mathrm{CO}_{2}$ results are used here. (The IVR rates are very uncertain, however.) This result provides indirect support for the conclusion that there is little contribution from $V-V$ transfer, yet it does not help to explain the deuterium isotope effect observed for the parent gas colliders. Perhaps the duration of collisions involving large polyatomic colliders is significantly longer than the straight line trajectories assumed in the simple model.

The origin of the unusual $\langle\langle E\rangle\rangle_{\mathrm{inv}}$ energy dependence observed for the deuterated species is not clear, but it could arise from resonance effects, if $V-V$ energy transfer is more important than was concluded in the preceding paragraphs. If the vibrational transition energies in the highly excited species are anharmonically shifted, as has been observed for the benzene C-H Htretching modes, ${ }^{36}$ the excited species fre- quencies will shift further out of resonance with the unexcited species as the energy of the former is increased, possibly reducing the efficiency of energy transfer. This explanation is highly conjectural and requires that $V-V$ energy transfer makes a significant contribution, contrary to the conclusions reached above. Until more definitive conclusions are reached about the mechanisms for energy transfer, this remains an open question.

More experiments are needed to determine the importance of $V-V$ energy transfer in collisions involving two polyatomics. It seems possible that the majority of energy transfer collisions involve $V \sim T / R$ transfer of relatively small energies, but a small fraction of collisions transfer significantly larger amounts of energy by $V-V$ transfer. If this conjecture is correct, $V-V$ energy transfer may contribute to "supercollisions" $41-43$ in which surprisingly large amounts of energy are transferred.

\section{ACKNOWLEDGMENTS}

This work was funded in part by the Department of Energy, Office of Basic Energy Sciences. Thanks go to J. D. Brenner for a careful reading of the manuscript and for discussions. J. R. B. thanks R. G. Gilbert for copies of papers prior to publication and for interesting discussions.

' For a recent review, see I. Oref and D. C. Tardy, Chem. Rev. 30, 1407 (1990).

2 (a) M. J. Rossi, J. R. Pladziewicz, and J. R. Barker, J. Chem. Phys. 78, 6695 (1983), and references therein; (b) J. R. Barker, J. Phys. Chem. 88, 11 (1984); (c) J. R. Barker and R. E. Golden, ibid. 88, 1012 (1984).

${ }^{3}$ J. Shi and J. R. Barker, J. Chem. Phys. 88, 6219 (1988); J. Shi, D. Bernfeld, and J. R. Barker, ibid. 88, 6211 (1988).

4 J. M. Zellweger, T. C. Brown, and J. R. Barker, J. Chem. Phys, 83, 6261 (1985).

${ }^{5}$ M. L. Yerram, J. D. Brenner, K. D. King, and J. R. Barker, J. Phys. Chem. 94, 6341 (1990).

${ }^{6}$ B. M. Toselli, J. D. Brenner, M. L. Yerram, W. E. Chin, K. D. King, and J. R. Barker, J. Chem. Phys. 95, 176 (1991).

${ }^{7}$ J. R. Barker, M. J. Rossi, and J. R. Pladziewicz, Chem. Phys. Lett. 90,99 (1982).

${ }^{8}$ B. M. Toselli and J. R. Barker, J. Chem. Phys. 95, 8108 (1991).

${ }^{9}$ B. M. Toselli, T. L. Walunas, and J. R. Barker, J. Chem. Phys. 92, 4793 (1990).

${ }^{10}$ A. Chimbayo, B. M. Toselli, and J. R. Barker (manuscript in preparation).

${ }^{11}$ H. Hippler and J. Troe, in Bimolecular Collisions, edited by M. N. R. Ashford and J. E. Baggott (Royal Society of Chemistry, London, 1989), p. 209.

"H. Hippler, L. Lindemann, and J. True, J. Chem. Phys. 83, 3906 (1985); H. Hippler, B. Otto, and J. Troe, Ber. Bunsenges. Phys. Chem. 93, 428 (1989).

${ }^{13}$ H. Hippler, J. Troe, and J. Wendelken, J. Chem. Phys. 78, 6709, 6718 (1983).

${ }^{14}$ M. Heymann, H. Hippler, D. Nahr, H. J. Plach, and J. Troe, J. Phys. Chem. 92, 5507 (1988).

15 J. E. Dove, H. Hippler, and J. Troe, J. Chem. Phys. 82, 1907 (1985); M. Heymann, H. Hippler, H. J. Plach, and J. Troe, ibid. 87, 3867 (1987).

${ }^{16}$ M. Damm, F. Deckert, H. Hippler, and J. Troe, J. Phys. Chem. 95, 2005 (1991).

17 W. Jalenak, R. E. Weston, Jr., T. J. Sears, and G. W. Flynn, J. Chem. Phys. 89, 2015 (1988).

${ }^{18}$ I. Z. Chou, S. A. Hewitt, J. F. Hershberger, B. B. Brady, G. B. Spector, L. Chid, and G. W. Flynn, J. Chem. Phys. 91, 5392 (1989); J. Z. Chou, S. A. Hewitt, J. F. Hershberger, and G. W. Flynn, ibid. 93, 8474 (1990).

${ }^{19}$ A. J. Sedlacek, R. E. Weston, and G. W. Flynn, J. Chem. Phys. 94, 6483 (1991). 
${ }^{20}$ (a) D. C. Tardy and B. S. Rabinovitch, Chem. Rev. 77, 369 (1977); (b) M. Quack and J. Troe, Gas Kinetics and Energy Transfer (Chemical Society, London, 1977), Vol. 2, p. 175.

${ }^{2}$ R. G. Gilbert and S. C. Smith, Theory of Unimolecular and Recombination Reactions (Blackwell Scientific, Oxford, 1990), Chap. 5.

${ }^{22}$ R. G. Gilbert, J. Chem. Phys. 80, 5501 (1984).

${ }^{23}$ K. F. Lim and R. G. Gilbert, J. Phys. Chem. 94, 72, 77 (1990).

${ }^{24}$ (a) K. F. Lim and R. G. Gilbert, J. Chem. Phys. 84, 6129 (1986); (b) K. F. Lim and R. G. Gilbert, ibid. 92, 1819 (1990).

${ }^{25}$ R. G. Gilbert and R. N. Zare, Chem. Phys. Lett. 167, 407 (1990).

${ }^{26}$ B. M. Toselli and J. R. Barker, Chem. Phys. Lett. 174, 304 (1990).

${ }^{27}$ B. J. Orr and I. W. M. Smith, J. Phys. Chem. 91, 6106 (1987).

${ }^{28}$ J. L. Knee, C. E. Ottis, and P. M. Juhnson, J. Chem. Phys. 81, 4455 (1984).

${ }^{29} \mathrm{~J}$. S. Swenton, in Isotopes in Organic Chemistry, edited by E. Buncel and C. C. Lee (Elsevier Scientific, Amsterdam, 1975), p. 241.

${ }^{30}$ T. E. Martin and A. H. Kalantar, Chem. Phys. Lett. 1, 623 (1968); P. M. Johnson and M. Studer, ibid. 18, 341 (1973).

${ }^{31}$ R. H. Page, R. H. Shen, and Y. T. Lee, J. Chem. Phys. 88, 5362 (1988); J. A. Draeger, Spectrochim. Acta 41, 607 (1985); H. D. Rudolph, H. Dreizler, A. Jauschke, and P. Wendling, Z. Naturforsch. 22, 940 (1967); N. Fuson, C. Garrigou-Lagrange, and M. L. Josien, Spectrochim. Acta 16, 106 (1960); T. Shimanouchi, Tables of Molecular Vibrational Fre- quencies, Vol. I, edited by NSRDS (1972); L. Goodman, A. G. Ozkabak, and S. N. Thakur, J. Phys. Chem. 95, 9044 (1991).

${ }^{32}$ G. Z. Whitten and B. S. Rabinovitch, J. Chem. Phys. 41, 1883 (1964).

${ }^{33}$ (a) W. Forst, Theory of Unimolecular Reactions (Academic, New York, 1973); (b) P. J. Robinson and K. A. Holbrook, Unimolecular Reactions (Wiley, New York, 1972), p. 137.

${ }^{34}$ T. C. Brown, K. D. King, and R. G. Gilbert, Int. J. Chem. Kinet. 20, 549 (1988).

${ }^{35}$ T. C. Brown, Ph.D. thesis, The University of Adelaide, Australia, 1988.

${ }^{36}$ J. D. Brenner and J. R. Barker, Astrophys. J. Lett. 388, L39 (1992).

${ }^{37}$ R. G. Gilbert (private communication, 1991).

${ }^{38}$ R. N. Schwartz, Z. I. Slawsky, and K. F. Herzfeld, J. Chem. Phys. 20, 1591 (1952).

${ }^{39}$ F. I. Tanczos, J. Chem. Phys. 30, 1119 (1959).

${ }^{40} \mathrm{~J}$. T. Yardley, Introduction to Molecular Energy Transfer (Academic, New York, 1980), Chap. 4.

${ }^{41}$ (a) S. Hassoon, I. Oref, and C. Steel, J. Chem. Phys. 89, 1743 (1988); (b) I. M. Morgulis, S. S. Sapers, C. Steel, and I. Oref, ibid. 90, 923 (1989); (c) A. Pashutzki and I. Oref, J. Phys. Chem. 92, 178 (1988).

${ }^{42}$ H. G. Löhmannsröben and K. Luther, Chem. Phys. Lett. 144, 473 (1988); K. Luther and K. Reihs, Rer. Bunsenges. Phys. Chem. 92, 442 (1988); K. Luther (private communication).

${ }^{43}$ G. Lendvay and G. C. Schatz, J. Phys. Chem. 94, 8864 (1990). 